\title{
DEUTSCHE FORSCHUNGSGEMEINSCHAFT FÖRDERT DEN AUFBAU EINES REPOSITORIUMS FÜR DIE GESCHLECHTER- FORSCHUNG
}

\section{von Karin Aleksander}

Die Deutsche Forschungsgemeinschaft hat Ende Januar 2016 einen Antrag aufFörderung des Aufbaus eines elektronischen Speicherortes für die Publikationen der Geschlechterforschung bewilligt.

Die elektronische Infrastruktur soll es ermöglichen, die Ergebnisse der Geschlechterforschung nachhaltig zu sichern und besser zugänglich zu machen. Gleichzeitig soll eine Plattform für freies Publizieren in einem fächerübergreifenden wissenschaftlichen Feld entstehen. Das Repositorium ermöglicht einen Brückenschlag zwischen der mehrere Jahrzehnte umfassenden Forschungsgeschichte der Frauen- und Geschlechterforschung und ihrer Zukunftsfähigkeit als anerkanntes und vernetztes Forschungsgebiet. Die Einrichtung des Repositoriums ist ein Schritt auf dem Weg zu einer an Open Access, dem offenen Zugang zu wissenschaftlichen Informationen, orientierten Publikationspraxis in der Geschlechterforschung. Mit seiner Hilfe soll das community-building für die Geschlechterforschung gestärkt, die Erschließung, Sicherung und Sichtbarmachung ihrer Wissensbestände befördert werden.

Beantragt wurde das Projekt von Dr. in Karin Aleksander, Zentrum für transdisziplinäre Geschlechterstudien an der Humboldt-Universität zu Berlin, Prof. ${ }^{\text {in }}$ Dr. ${ }^{\text {in }}$ Sabine Hark, Zentrum für interdisziplinäre Frauen- und Geschlechterforschung an der Technischen Universität Berlin, sowie Dr. ${ }^{\text {in }}$ Anita Runge, Margherita-von-Brentano-Zentrum für Geschlechterforschung an der Freien Universität Berlin.

Die Deutsche Forschungsgemeinschaft fördert das Projekt mit drei Vollzeitstellen für wissenschaftliche Mitarbeiter_innen über zwei Jahre mit rund 500.000 Euro.

Die Projektgruppe freut sich über Interesse an Kooperation und lädt Interessierte ausdrücklich ein, sich mit ihren Publikationen am Aufbau des Repositoriums zu beteiligen.

\section{Kontaktpersonen:}

- Karin Aleksander, HU Berlin (karin.aleksander@gender.hu-berlin.de),

- Sabine Hark, TU Berlin (sabine.hark@tu-berlin.de),

- Anita Runge, FU Berlin (anita.runge@fu-berlin.de). 


\section{Dr. ${ }^{\text {in }}$ Karin Aleksander}

Zentrum für transdisziplinäre Geschlechterstudien an der Humboldt-Universität zu Berlin

Georgenstraße 47, D-10117 Berlin E-Mail: karin.aleksander@gender.hu-berlin.de

Dieses Werk ist lizenziert unter einer

Creative-Commons-Lizenz Namensnennung 4.0 International 